Cell research (2003); 13(6):503-507

http://www.cell-research.com

SHORT COMMUNICATION

\title{
Mortalin imaging in normal and cancer cells with quantum dot immuno- conjugates
}

\author{
Zeenia KAUL, Tomoko YAGUCHI, Sunil C KAUL, Takashi HIRANO, Renu WADHWA*, Kazunari TAIRA
}

National Institute of Advanced Industrial Science and Technology (AIST), 1-1-1 Higashi, Tsukuba, Ibaraki 3058562, Japan E-mail: renu-wadhwa@aist.go.jp

\begin{abstract}
Quantum dots are the nanoparticles that are recently emerging as an alternative to organic fluorescence probes in cell biology and biomedicine, and have several predictive advantages. These include their i) broad absorption spectra allowing visualization with single light source, ii) exceptional photo-stability allowing long term studies and iii) narrow and symmetrical emission spectrum that is controlled by their size and material composition. These unique properties allow simultaneous excitation of different size of quantum dots with a single excitation light source, their simultaneous resolution and visualization as different colors. At present there are only a few studies that have tested quantum dots in cellular imaging. We describe here the use of quantum dots in mortalin imaging of normal and cancer cells. Mortalin staining pattern with quantum dots in both normal and cancer cells mimicked those obtained with organic florescence probes and were considerably stable.
\end{abstract}

Key words: quantum dots, immunofluorescence, mortalin, visualization.

\section{INTRODUCTION}

Nano is a Greek prefix that defines the smallest (1000 times smaller than micrometer) natural structures and nanotechnology, a most recent and highly energized discipline of science and technology that deals with manipulation and use of these structures with superior electrical, chemical, mechanical or optical properties. Applications of nanostructures (quantum dots) have recently been extended to biology (nanobiology), biotechnology (nanobiotechnology) and biomedicine (nanomedicine). Their speculated use extends from disease diagnosis to therapy. For example, presence of a molecule or disease causing organisms can be detected by nanotagged antibodies. In this approach, antibodies specific to a disease causing protein labeled with magnetic nanoparticles are subjected to brief magnetic field. Bound antibodies give off a strong magnetic signal whereas

"Corresponding author: Renu WADHWA, Gene Function Research Center, National Institute of Advanced Industrial Science and Technology (AIST), 1-1-1 Higashi, Tsukuba, Ibaraki 305-8562, Japan Tel: +8129861 9464, Fax: +81298613019

E-mail: renu-wadhwa@aist.go.jp

Received Sept-6-2003 Revised Sep-15-2003 Accepted Sep-25-2003 unbound antibodies stumble in all directions and produce no net signal[1,2], thus the presence of a disease specific protein can be easily detected. At DNA level, short DNA segments with disease specific sequences can be tagged with gold nanoparticles and can possibly be further used for easy detection of complementary sequences in specimens[3-5]. A more dramatic potential application of nanotechnology is the use of nanoshells in cancer therapy, in which tumor cell specific antibodies can be linked to nano-gold plated spheres (nanoshells, extremely small beads of glass coated with gold)[6]. Nanoshells can be used to deliver drug molecules at specific times by attaching it to capsule made of a heat sensitive polymer. The capsule would release its contents only when gentle heating of the attached nanoshells cause it to deform. Such heating of nanoshells in the body should destroy the cancer cells, while leaving nearby tissue unharmed [7-9]. Other types of nanostructures possessing high surface area may prove useful for delivering drugs and DNA for gene therapy[10-12]. They might be safer than other leading method including genetically modified viruses. Similarly, biologically compatible nanoparticles if engineered successfully may serve as more stronger and 
durable tissue implants[13, 14]. A recent exciting application of nanobiology has emerged as the use of quantum dots in cellular imaging[15-17]. Quantum dots are the latex beads. As their name suggests, they owe their special properties to the rules of quantum mechanics that restrict the electrons in atoms to certain discrete energy levels. Like bulk semiconductors, they absorb photons of all energies above the threshold of the band gap. However, the wavelength of the light quantum dot emits (in other words, its color) depends largely on its size. Hence a simple semi-conducting material can yield an entire family of distinctly colored labels.

Recently, these luminescent quantum dots have been developed as an alternative to organic dyes for fluorescence based application in biology and medicine[15-17]. Biological systems are very complex, and at most cases different components must be observed simultaneously. Such tracking is difficult to achieve, because each organic dye must be excited with different wavelength. With quantum dots, it is possible to tag a variety of biological molecules, each with a crystal of different size (hence a different color) and because all of these crystals can be energized with a single light source, they can all be monitored at once. These inorganic crystals have several advantages over conventional organic dye molecules. For example they are very stable. They can withstand much more cycles of excitation and light emission than typical organic molecules, which soon get bleached. Furthermore, these quantum dots depending on their sizes can emit a wide range of colors with a single excitation light source (Fig 1). These features make it possible that not only multiple cells could be imaged at the same time but could be followed for longer intervals. Thus these nanoscale semiconductors can potentially serve as unique labels for biomolecules. Quantum dot bioconjugates are made from a nanometer scale crystal of semiconductor material, cadmium selenide, which is coated with an additional semiconductor shell $(\mathrm{ZnS})$ to improve the optical properties of the material[16]. This core shell material is further coated with polymer shell or other ligands that allows these materials to be conjugated to the biomolecules and to retain their optical properties. The polymer shell can be directly coupled to streptavidin or other biomolecules. In the present study, we compared the immunofluore-scent images of a heat shock 70 family protein, mortalin, using conventional organic fluorescence dye with quantum dot conjugates.

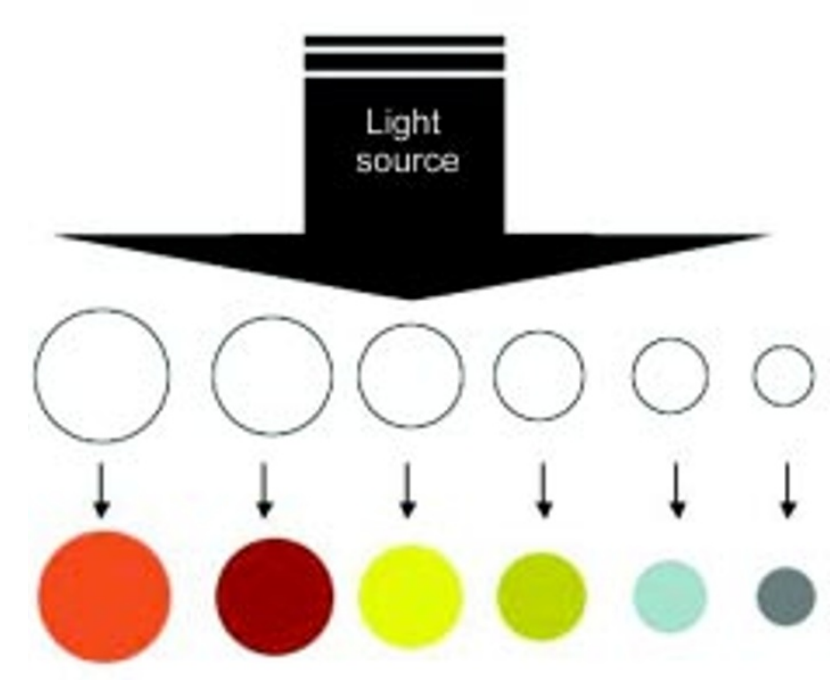

Fig 1 Diagrammatic presentation of quantum dot size and their emission spectrum yielding colors. Nanocrystals absorb light and then re-emit the light in a different color. The different colors are determined by the size of the nanocrystals.

\section{MATERIAL AND METHODS}

Normal human foetal fibroblasts (WI-38) and osteogenic sarcoma (U2OS) cells were cultured in Dulbecco's modified Eagle's minimal essential medium (DMEM) supplemented with $10 \%$ fetal bovine serum (FBS), penicillin, streptomycin and fungizone (Life Technologies, Inc.). For indirect immunofluorescence studies, cells were plated on glass coverslips placed in 12-well culture dish at densities ranging from $10^{3}$ to $10^{4}$ cells per coverslip. After $24 \mathrm{~h}$, when cells had attached to the surface and spread well, they were washed with cold phosphate buffered saline (PBS), and then fixed with pre-chilled methanol/acetone $(1 / 1, \mathrm{v} / \mathrm{v})$ mixture for $5 \mathrm{mins}$ on ice. Fixed cells were washed with PBS, permeabilized with $0.5 \%$ Triton X-100 in PBS for 15 mins, and blocked with blocking reagent (Sigma) for $1 \mathrm{~h}$. They were incubated with anti-mortalin antibodies (monoclonal anti-mthsp70; Affinity Bioreagents, and polyclonal antimortalin antibody[18]) for $1 \mathrm{~h}$ at room temperature, washed thrice with $0.2 \%$ Triton X-100 in PBS, and then incubated with secondary antibodies (rabbit Alexa 488)(Molecular Probes); Alexa Fluor ${ }^{\circledR} 488$ goat anti-rabbit $\operatorname{IgG}(\mathrm{H}+\mathrm{L})$ and mouse biotin (goat anti-mouse IgGB) for 30 mins, followed by washings with $0.2 \%$ Triton $\mathrm{X}-100$ in PBS. For quantum dots staining, cells were incubated with QD (Qdot ${ }^{\mathrm{TM}} 605$ Streptavidin Conjugate; Qantum Dot Corporation, USA) in QD incubation Buffer (1:2000). After three washings of $10 \mathrm{mins}$ each with $0.2 \%$ Triton X-100 in PBS and a final wash with PBS, the coverslips were mounted on glass slides with Fluoromount (Difco).

\section{RESULTS AND DISCUSSION}

Mortalin is a member of hsp70 family of proteins. It locates at multiple subcellular sites and shows differential staining pattern in human normal and transformed cells. Whereas normal human cells show uniform pancytoplasmic 
staining, transformed human cells exhibit perinuclear staining pattern[19]. Such differential subcellular staining of mortalin has been suggested as a reliable marker for normal and transformed cells[20,21]. Another important aspect of mortalin is its functional properties in normal and transformed cells. It has been shown to interact with many other proteins[22, 23]. The functional significance of its interactions to the binding partners in different cellular phenotypes and in response to stress conditions has not been well studied. Such studies on subcellular distribution of mortalin and its functional aspects in normal and transformed cells require visualization tools such as specific antibodies. Conventional immunofluorescence techniques involve the staining with the first antibody and visualization by secondary staining with either fluorescein isothiocyanate (FITC) or Texas Red or Alexa-conjugated IgG etc. These organic fluorescent dyes require UV light source, undergo photobleaching and have wide emission range so that multiple protein staining images cannot be observed simultaneously. Thus quantum dots (Qdots) offer a practical alternative to the conventional organic fluorescent dyes[15, 16]. We used Qdot ${ }^{\mathrm{TM}} 605$ Streptavidin conjugate for mortalin staining in normal and transformed cells. As shown in Fig 2, we obtained mortalin-staining patterns that have been reported previously by using fluorescent dyes[19]. Whereas normal human cells showed uniform staining in the cytoplasm, the transformed (U2OS) cells showed a perinuclear pattern. Mortalin has been shown to occur in multiple subcellular sites[24]. The above results

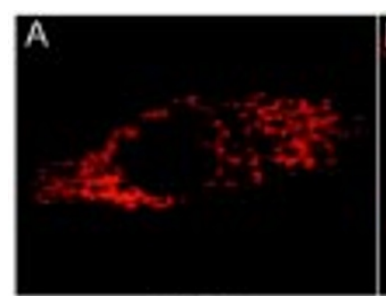

Wh-38

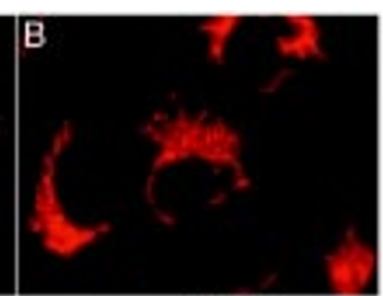

U2OS
Fig 2 Quantum dot mortalin staining patterns in normal (embryonic fibroblasts, WI-38) (A) and cancer (osteocarcinoma, U2OS) (B) human cells.

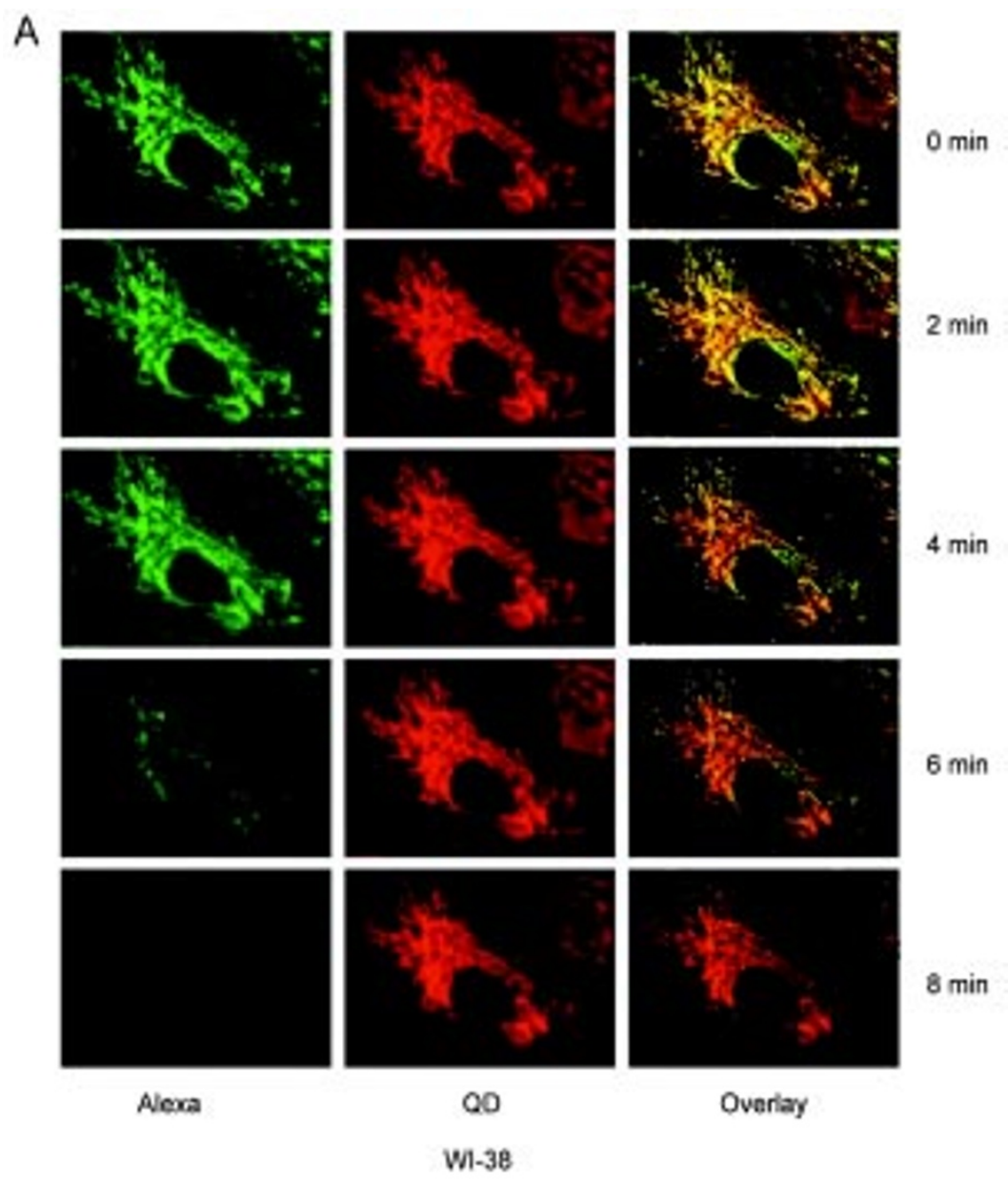

Fig 3A Immunofluorescence images of mortalin to compare the photostability between Alexa 488 and quantum dots in normal cells (Details as in Fig 3B). suggested that the quantum dots could be used for multicolor visualization of proteins at any subcellular site.

We next analyzed the stability of quantum dot staining versus fluorescence staining. Normal (WI-38) and cancer derived (U2OS) human cells were stained for mortalin and visuali-zed by Alexa and Qdots. This allowed simultaneous evaluation of their signal patterns and stabilities under same conditions. Stained cells were illuminated with light form low mercury lamp and were photographed. As shown in Figs 3A and 3B, fluorescence staining with Alexa probe started to fade away after 3-4 mins of exposure both in normal and transformed cells and was completely abolished after 8 mins. Such limitations of fluorescent dyes can lead to errors in the visualization studies related to subcellular localization and their functional relevance in terms of interactions with other pro- 
teins. On the contrary, Qdots-mortalin staining did not fade away with time (Figs $3 \mathrm{~A}$ and 3B). Such stable staining of proteins is of practical value for structural, temporal, interactive and functional studies.

\section{ACKNOWLEDGEMENTS}

The study was partly supported by grants from the New Energy and Industrial Technology Development Organization (NEDO), Japan.

\section{REFERENCES}

$1 \mathrm{Gu}$ H, Ho PL, Tsang KW, Yu CW, Xu B. Using biofunctional magnetic nanoparticles to capture gram-negative bacteria at an ultra-low concentration. Chem Commun (Camb), 2003; 15: 966-7.

2 Perez JM, Simeone FJ, Saeki Y, Josephson L, Weissleder R. Viral-induced self-assembly of magnetic nanoparticles allows the detection of viral particles in biological media. J Am Chem Soc 2003; 125:10192-3.

3 Lee HY, Sacho Y, Kanki T, Tanaka H, Shirakawa H, Cheon JW, Yoon JH, Kang NJ, Park JI, Kawai T. DNA-directed magnetic network formations with ferromagnetic nanoparticles. J Nanosci Nanotechnol 2002; 2:613-5.

4 Nakayama H, Arakaki A, Maruyama K, Takeyama H, Matsunaga T. Single-nucleotide polymorphism analysis using fluorescence resonance energy transfer between DNA-labeling fluorophore, fluorescein isothiocyanate, and DNA intercalator, POPO-3, on bacterial magnetic particles. Biotechnol Bioeng 2003; 84: 96-102.

5 Stevenson KA, Muralidharan G, Maya L, Wells JC, Barhen J, Thundat T. Covalent attachment of gold nanoparticles to DNA templates. J Nanosci Nanotechnol 2002; 2:397-404.

6 Brongersma ML. Nanoscale photonics: Nanoshells: gifts in a gold wrapper. Nat Mater 2003; 2:296-7.

7 Harisinghani MG, Barentsz J, Hahn PF, DesernoWM, Tabatabaei S, van de $\mathrm{Kaa} \mathrm{CH}$, de la Rosette J, Weissleder R. Noninvasive detection of Weissleder R. Noninvasive detection of clinically occult lymphnode meta-stases in prostate cancer. N Engl J Med 2003; 348: 2491-9.

8 Pitsillides CM, Joe EK, Wei X, Anderson RR, Lin CP. Selective cell targeting with light-absorbing microparticles and nanoparticles. Biophys J 2003; 84:4023-32.

9 Sokolov K, Follen M, Aaron J, Pavlova I, Malpica A, Lotan R, Richards-Kortum R. Real-time vital optical imaging of precancer using anti-epidermal growth factor receptor antibodies conjugated to gold nanoparticles. Cancer Res 2003; 63: 1999-2004.

10 Emerich DF, Thanos CG. Nanotechnology and medicine. Expert Opin Biol Ther 2003; 3:655-63.
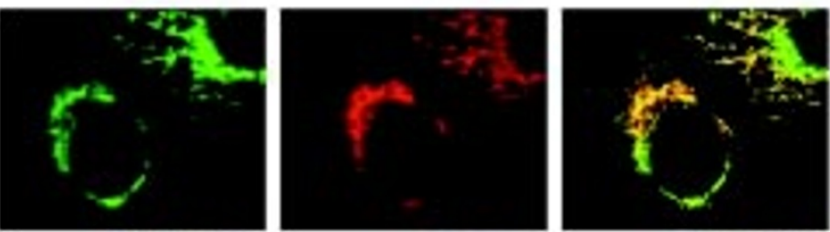

$0 \mathrm{~min}$
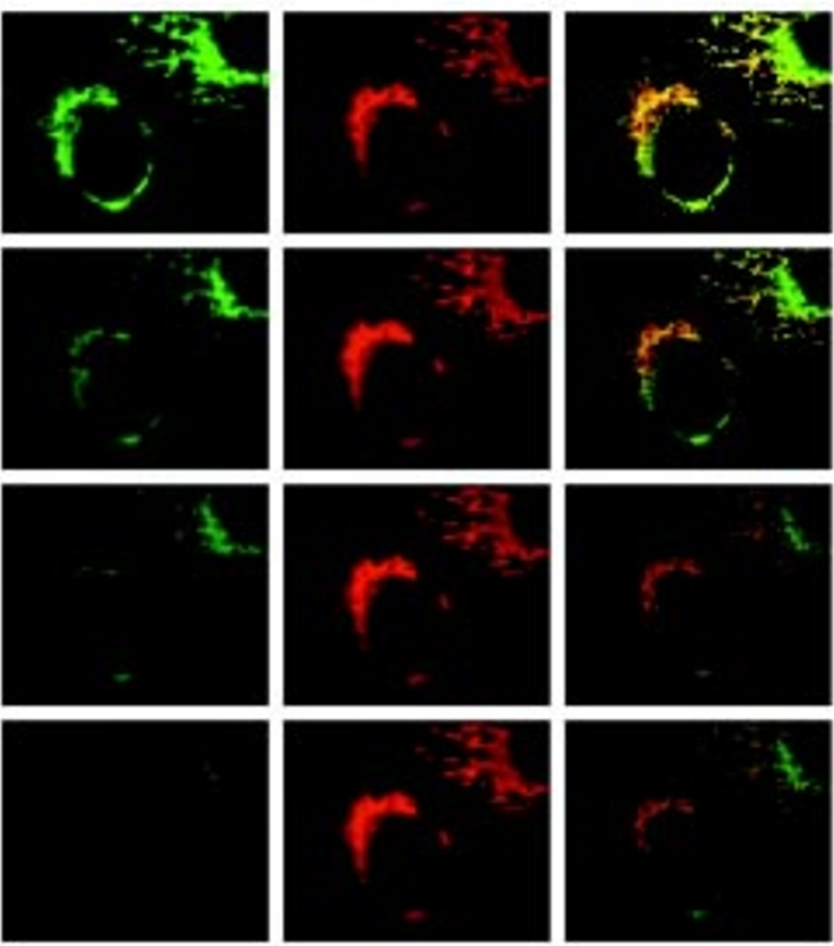
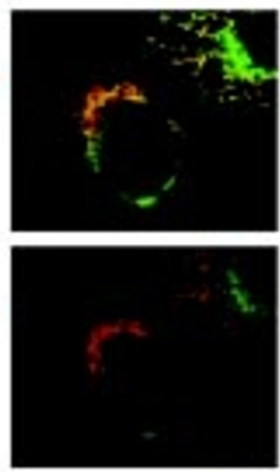

$6 \mathrm{~min}$

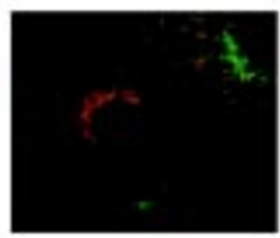

Overlay

$2 \mathrm{~min}$

$4 \mathrm{~min}$

$8 \mathrm{~min}$

\section{u2Os}

Fig 3B Immunofluorescence images of mortalin to compare the photostability between Alexa 488 and quantum dots in transformed cells. The specimens were continuously illuminated for $9 \mathrm{~min}$ with light from a $100 \mathrm{~W}$ mercury lamp under a $100 \times 1.30$ oil-immersion objective. Images were captured with a cooled CCD camera at $1 \mathrm{~min}$ intervals for each color. When labeling signals of Alexa 488 started fading and became undetectable in both kinds of cells, the signals of Qds showed no obvious change for the entire 9 min illumination period.

11 Menei P, Benoit JP, Boisdron-Celle M, Fournier D, Mercier P, Guy G. Drug targeting into the central nervous system by stereotactic implantation of biodegradable microspheres. Neuro-surgery $1994 ; 34: 1058-64$.

12 Panyam J, Labhasetwar V. Biodegradable nanoparticles for drug and gene delivery to cells and tissue. Adv Drug Deliv Rev 2003; 55:329-47.

13 Curtis A, Wilkinson C. Nantotechniques and approaches in biotechnology. Trends Biotechnol 2001; 19:97-101.

14 Prokop A. Bioartificial organs in the twenty-first century: nanobiological devices. Ann N Y Acad Sci 2001; 944:472-90.

15 Jaiswal JK, Mattoussi H, Mauro JM, Simon SM. Long-term multiple color imaging of live cells using quantum dot bioconjugates. Nat Biotechnol 2003; 21:47-51. 
16 Watson A, Wu X, Bruchez M. Lighting up cells with quantum dots. Biotechniques 2003; 34: 296-300.

$17 \mathrm{Wu}$ X, Liu H, Liu J, Haley KN, Treadway JA, Larson JP, Ge $\mathrm{N}$, Peale F, Bruchez MP. Immunofluorescent labeling of cancer marker Her2 and other cellular targets with semiconductor quantum dots. Nat Biotechnol 2003; 21:41-6.

18 Wadhwa R, Kaul SC, Ikawa Y, Sugimoto Y. Identification of a novel member of mouse hsp70 family. J Biol Chem 1993; 268: 6615-21.

19 Wadhwa R, Kaul SC, Mitsui Y, Sugimoto Y. Differential subcellular distribution of mortalin in mortal and immortal mouse and human fibroblasts. Exp Cell Res 1993; 207:442-48.

20 Michishita E, Nakabayashi K, Suzuki T, Kaul SC, Ogino H, Fujii M, Mitsui Y, Ayusawa D. 5-Bromodeoxyuridine induces senescence-like phenomena in mammalian cells regardless of cell type or species. J Biochem 1999; 126:1052-9.

21 Wadhwa R, Sugihara T, Yoshida A, Nomura H, Reddel RR, Simpson R, Maruta H, Kaul SC. Selective toxicity of MKT077 to cancer cells is mediated by its binding to the hsp70 family protein mot- 2 and reactivation of $\mathrm{p} 53$ function. Cancer Res 2000; 60:6818-21.

22 Wadhwa R, Taira K, Kaul SC. An hsp70 family chaperone, mortalin/mthsp70/PBP74/Grp75: what, when and where? Cell Stress \& Chaperones 2002; 7:309-16.

23 Kaul SC, Taira K, Pereira-Smith OM, Wadhwa R. Mortalin: present and prospective. Exp Gerontol 2002; 37: 1157-1164.

24 Ran Q, Wadhwa R, Kawai R, Kaul SC, Sifers RN, Bick RJ, Smith JR, Pereira-Smith OM. Extramitochondrial localization of mortalin/mthsp70/PBP74/GRP75. Biochem Biophys Res Commun 2000; 275:174-9. 